\title{
AN INTEGRAL RIEMANN-ROCH FORMULA FOR INDUCED REPRESENTATIONS OF FINITE GROUPS
}

BY

\author{
LEONARD EVENS AND DANIEL S. KAHN ${ }^{1}$
}

\begin{abstract}
Let $\boldsymbol{H}$ be a subgroup of the finite group $G, \boldsymbol{\xi}$ a finite dimensional complex representation of $H$ and $\rho$ the induced representation of $G$. If $s_{k}(\rho) \in H^{2 k}(G, Z), k>1$, denote the characteristic classes bearing the same relation to power sums that Chern classes bear to elementary symmetric functions, then we prove the following,
\end{abstract}

$$
\bar{N}(k)\left(s_{k}(\rho)-\operatorname{Tr}_{H \rightarrow G}\left(s_{k}(\xi)\right)\right)=0,
$$

where

$$
\bar{N}(k)=\prod_{\substack{p \mid N(k) \\ p \text { prime }}} p
$$

and

$$
N(k)=\left(\prod_{p \text { prime }} p^{[k / p-1]}\right) / k ! .
$$

(Tr denotes transfer.) Moreover, $\bar{N}(k)$ is the least integer with this property.

This settles a question originally raised in a paper of Knopfmacher in which it was conjectured that the required bound was $N(k)$.

1. Introduction. Let $G$ be a finite group, and $\rho: G \rightarrow U_{n}$ a unitary representation of degree $n$. Various "characteristic classes" have been defined for $\rho$. The Chern classes $c_{i}(\rho) \in H^{2 i}(G, \mathrm{Z}), 0 \leqslant i \leqslant n$, were introduced by Atiyah in [At]. Other classes, $s_{k}(\rho) \in H^{2 k}(G, \mathrm{Z}), 1<k<\infty$, which bear the same relation to power sums that Chern classes bear to elementary symmetric functions, were first studied in the context of group cohomology by Knopfmacher [Kn] and were also investigated by C. B. Thomas ([T1] and [T2]). Knopfmacher showed that for $\xi: H \rightarrow U_{n}$ a representation of a subgroup $H<G$, the class $s_{k}(\rho)$, where $\rho=\operatorname{ind}_{H \rightarrow G} \xi$ (the induced representation), satisfies

$$
N_{k}\left(s_{k}\left(\text { ind }_{H \rightarrow G} \rho\right)-\operatorname{Tr}_{H \rightarrow G}\left(s_{k}(\rho)\right)\right)=0
$$

Received by the editors April 28, 1976.

AMS (MOS) subject classifications (1970). Primary 20C00, 55F40: Secondary $18 \mathrm{H} 10$.

Key words and phrases. Characteristic class, Chern class, Knopfmacher, Riemann-Roch formula, induced representation, group, transfer, wreath product.

${ }^{1}$ The research reported on in this paper was supported by NSF Grants MPS71-02912 A05 (first author) and MPS75-06976 (second author). 
where $N_{k}$ is a (large) integer depending only on $k$ and not on the group, subgroup, or representation. Knopfmacher asserts "that heuristic considerations (suggested ... by J. F. Adams and based on Riemann-Roch theorems) make it conceivable that the least positive integer $N_{k}$ with the property ... is the number

$$
N(k)=(k !)^{-1} \prod_{\text {prime } p} p^{[k / p-1]}
$$

It is our purpose here to establish a modified form of this conjecture. Namely, define

$$
\bar{N}(k)=\prod_{\substack{p \text { prime } \\ p \mid N(k)}} p
$$

so that $\bar{N}(k)$ contains exactly those primes occurring in $N(k)$ but to the first power. Then, we shall demonstrate that $\bar{N}(k)$ is the least positive integer $N_{k}$ such that $(\mathrm{K})$ holds for all finite $G, H$, and $\xi$.

Our approach is computational, and we must admit that the number $\bar{N}(k)$ does not arise naturally from the argument. The reader might also find enlightening earlier partial results on the problem in [T1] and [T2]. (After we completed this work, J. C. Becker brought to our attention the Princeton thesis of F. Roush [1971] in which the modified conjecture was also proved. Roush's methods are substantially independent of ours.)

Before proceeding, we should note that the classes $s_{k}(\rho)$ seem to contain relatively little information, at least compared to the Chern classes. In \$4, we work out a few examples which seem to support our pessimism about the utility of these classes. (See [Ch1] and [Ch2] for what we feel are much stronger results about the Chern classes of induced representations. There is some overlap between [Ch2] and this paper.)

2. The case of $P \int T$. We recall a few simple facts about the classes $s_{k}(\rho)$, $k=0,1,2, \ldots$ (In what follows, "group" generally means "finite group," but in some cases "compact Lie group" is an allowed meaning. "Representation" means "finite dimensional unitary representation.") A good background source is $[\mathbf{H}]$.

(a) If $\rho$ and $\xi$ are representations of the group $G$, then, for each $k>0$,

$$
s_{k}(\rho \oplus \xi)=s_{k}(\rho)+s_{k}(\xi) \text {. }
$$

(b) If $\rho$ is a representation of $G$ and $\phi: H \rightarrow G$ is a homomorphism, then, for each $k>0$,

$$
s_{k}(\rho \circ \phi)=\phi^{*}\left(s_{k}(\rho)\right) \text {. }
$$

(c) Let $\iota: T^{n} \rightarrow U_{n}$ be the inclusion of the $n$-dimensional torus in $U_{n}$. Writing, as usual, $H^{*}\left(B T^{n}, \mathbf{Z}\right)=\mathbf{Z}\left[x_{1}, \ldots, x_{n}\right]$, we have 


$$
s_{k}(\iota)=x_{1}^{k}+x_{2}^{k}+\cdots+x_{n}^{k} .
$$

Notice that, for our purposes, we may essentially use (b) and (c) to define $s_{k}(\rho)$. Namely,

$$
H^{*}\left(B U_{n}, \mathbf{Z}\right)=\mathbf{Z}\left[C_{1}, \ldots, C_{n}\right] \rightarrow H^{*}\left(B T^{n}, \mathbf{Z}\right)=\mathbf{Z}\left[x_{1}, \ldots, x_{n}\right]
$$

is a monomorphism onto the subring of symmetric polynomials. $\left(C_{i} \mapsto \phi_{i}=\right.$ the $i$ th elementary symmetric function.) Since $x_{1}^{k}+\cdots+x_{n}^{k}$ is symmetric, it has a unique pre-image, $S_{k} \in H^{2 k}\left(B U_{n}, \mathbf{Z}\right)$. Then, because of (b), given $\rho$ : $G \rightarrow U_{n}$,

$$
s_{k}(\rho)=\rho^{*}\left(S_{k}\right) .
$$

The relations between Chern classes $c_{i}$ and the classes $s_{k}$ are given by the so-called Newton formulas. For our purposes, it will be useful to express these as follows. Write $p_{k}=x_{1}^{k}+\cdots+x_{n}^{k}$. The usual derivation, [W, Chapter II, §3, p. 38], adapted to a calculation in the ring of formal power series $\mathbf{Z}\left[\left[x_{1}, \ldots, x_{n}\right]\right]$ yields

$$
\sum_{k=1}^{\infty}(-1)^{k+1} p_{k}=\frac{\phi^{\prime}}{\phi}
$$

where $\phi=1+\phi_{1}+\phi_{2}+\cdots+\phi_{n}=\prod_{i=1}^{n}\left(1+x_{i}\right)$ and $\phi^{\prime}=\phi_{1}+2 \phi_{2}$ $+\cdots+n \phi_{n}$. Because of the monomorphism (B), and (Df) above, this translates, for $\rho: G \rightarrow U_{n}$ into the formula

$$
\sum_{k=1}^{\infty}(-1)^{k+1} s_{k}(\rho)=\frac{c^{\prime}(\rho)}{c(\rho)}
$$

where $c(\rho)=1+c_{1}(\rho)+\cdots+c_{n}(\rho)$ and $c^{\prime}(\rho)=c_{1}(\rho)+2 c_{2}(\rho)+\cdots+$ $n c_{n}(\rho)$. (The calculation should take place in $\Pi_{k=0}^{\infty} H^{2 k}(B G, Z)$. It makes sense since $c(\rho)=1+c_{1}+\cdots+c_{n}$ is invertible there.)

In what follows, we shall make free use of the results on wreath products in [N] and [Ch1]; also, we use the terminology and notation of those papers. If $S$ is any subgroup of $S_{n}$, we may form the wreath product, $S \int T=S \cdot T^{n}$, and this is a subgroup of $U_{n}$. Since $H^{*}(B T, \mathbf{Z})=\mathbf{Z}[x]$ is $\mathbf{Z}$-free, there is an isomorphism

$$
H^{*}\left(B\left(S \int T\right), \mathrm{Z}\right) \cong H^{*}\left(S, H^{*}\left(B T^{n}, \mathrm{Z}\right)\right)
$$

which we call the Nakaoka isomorphism. (Nk) is a ring isomorphism, and it is natural with respect to changes in the subgroup $S$ (for restriction or transfer). (See [Ch1, \&2, pp. 181-183] for details.) Below, we are interested in the special case $n=p$ is prime, and $S=P$ is cyclic of order $p$ (say generated by the cycle $(123 \ldots p))$. Thus,

$$
H^{*}(B(P S T), \mathrm{Z}) \cong H^{*}\left(P, \mathrm{Z}\left[x_{1}, x_{2}, \ldots, x_{p}\right]\right)
$$

where $P$ permutes the variables cyclically. 
As a $P$-module, each homogeneous component of $\mathbf{Z}\left[x_{1}, \ldots, x_{p}\right]$ breaks up into a direct sum of submodules either (i) isomorphic to $\mathbf{Z}[P]$ or (ii) isomorphic to $\mathbf{Z}$. The second type are all of the form $\mathbf{Z} \phi_{p}^{j}$ where $\phi_{p}=$ $x_{1} x_{2} \ldots x_{p}$. In particular, for degrees $1,2, \ldots, p-1$ (in the $x_{i}$ ), only type (i) submodules occur. Since $H^{r}(P, Z[P])=0, r>0$, we can prove

Lemma. The total Chern class of the inclusion $\iota^{\prime}: P \int T \rightarrow U_{p}$ is given by

$$
c\left(\iota^{\prime}\right)=\phi-\mu^{p-1}
$$

where $\mu$ generates $H^{2}(P, \mathrm{Z})$.

Proof. In essence, this is proved in [Ch1, Theorem 4 and Corollary 5]. However, it follows easily from the above remark and identification of the components of the Chern classes on the "edges", $H^{0}\left(P, \mathrm{Z}\left[x_{1}, \ldots, x_{n}\right]\right)$ and $H^{*}(P, \mathrm{Z})$. (See [Ch1, §3, p. 185].)

Note. See also [Ch2, Lemma in Theorem I] where the same lemma plays a role.

We shall now derive the crucial formula.

Proposition I. Write $s_{k}=s_{k}\left(\iota^{\prime}\right)$. Then, with the notation as above,

$$
\begin{aligned}
\sum_{k=1}^{\infty}(-1)^{k+1}\left(s_{k}-p_{k}\right) & =\frac{\mu^{p-1}}{1-\mu^{p-1}+\phi_{p}} \\
& =\sum_{n=0}^{\infty} \mu^{p-1}\left(\mu^{p-1}-\phi_{p}\right)^{n} .
\end{aligned}
$$

Note. For $k=1,2, \ldots, p-2$, the above formula yields $s_{k}=p_{k}$, and for $k=p-1, p$,

$$
s_{p-1}=p_{p-1}-\mu^{p-1}, \quad s_{p}=p_{p} .
$$

These latter results were obtained by Thomas in [T1], also by means of Newton's formulas.

Proof of Proposition. By (Nt2), we have

$$
\sum_{k=1}^{\infty}(-1)^{k+1} s_{k}=\frac{c^{\prime}}{c} \text {. }
$$

Also, on the "edge", $H^{0}\left(P, \mathrm{Z}\left[x_{1}, \ldots, x_{n}\right]\right)$,

$$
\sum_{k=1}^{\infty}(-1)^{k+1} p_{k}=\frac{\phi^{\prime}}{\phi} \text {. }
$$

By the lemma,

$$
c=\phi-\mu^{p-1}, \quad c^{\prime}=\phi^{\prime}-(p-1) \mu^{p-1}=\phi^{\prime}+\mu^{p-1}
$$

since $p \mu=0$. Thus, 


$$
\begin{aligned}
\sum_{k=1}^{\infty}(-1)^{k+1}\left(s_{k}-p_{k}\right) & =\frac{\phi^{\prime}+\mu^{p-1}}{\phi-\mu^{p-1}}-\frac{\phi^{\prime}}{\phi} \\
& =\frac{\phi^{\prime} \phi+\mu^{p-1} \phi-\phi \phi^{\prime}+\phi^{\prime} \mu^{p-1}}{\phi\left(\phi-\mu^{p-1}\right)} \\
& =\frac{\phi \mu^{p-1}+\phi^{\prime} \mu^{p-1}}{\phi\left(\phi-\mu^{p-1}\right)}
\end{aligned}
$$

(Note that all this makes sense since $\phi$ and $\phi-\mu^{p-1}$ are invertible in $\prod_{k=0}^{\infty} H^{2 k}\left(B\left(P \int T\right), Z\right)$.)

To reduce this further, we must say something about the product structure in $H^{*}\left(P, \mathbf{Z}\left[x_{1}, \ldots, x_{p}\right]\right)$. Given $\alpha \in H^{r}(P, Z), r>0$, and $\beta \in H^{0}(P, M)=$ $M^{P}$, where $M$ is a direct summand of $\mathbf{Z}\left[x_{1}, \ldots, x_{p}\right]$ (as described above), then $\alpha \beta$ may be identified with an element of $H^{r}(P, M)$, which is in turn a direct summand of $H^{r}\left(P, \mathbf{Z}\left[x_{1}, \ldots, x_{p}\right]\right)$. In particular, if $M \cong \bigoplus Z[P]$, then $\alpha \beta=0$, whatever $\alpha$ may be. Thus, the following products are trivial.

\begin{tabular}{c|c} 
Product & P-Module \\
\hline$\mu^{p-1} \phi_{j}=0, \quad j=1,2, \ldots, p-1$ & $\underset{\begin{array}{c}i_{1}, \ldots, i_{j} \\
\text { distinct }\end{array}}{\left.\mathbf{Z}_{i_{1}} x_{i_{2}} \cdots x_{i_{j}} \cong \mathbf{Z}[P]\right]_{j}^{(p) / p}}$ \\
\hline$\mu^{p-1} p_{k}=0, \quad k=1,2, \ldots$ & $\mathbf{Z} x_{1}^{k}+\mathbf{Z}_{2}^{k}+\cdots+\mathbf{Z}_{p}^{k} \cong \mathbf{Z}[P]$.
\end{tabular}

By an analogous argument, $\mu^{i} \phi_{p}^{j} \neq 0$ since $M=\mathbf{Z} \phi_{p}^{j} \cong \mathbf{Z}$ as a $P$-module.

We may now calculate further.

$$
\begin{aligned}
\mu^{p-1} \phi^{\prime} & =\mu^{p-1}\left(\phi_{1}+2 \phi_{2}+\cdots+p \phi_{p}\right) \\
& =p \mu^{p-1} \phi_{p}=0 .
\end{aligned}
$$

Hence,

$$
\sum_{k=1}^{\infty}(-1)^{k+1}\left(s_{k}-p_{k}\right)=\frac{\phi \mu^{p-1}}{\phi\left(\phi-\mu^{p-1}\right)}=\frac{\mu^{p-1}}{\phi-\mu^{p-1}} .
$$

Write $\phi=1+\dot{\phi}$. Then, the above quotient may be expanded

$$
\frac{\mu^{p-1}}{1-\left(\mu^{p-1}-\dot{\phi}\right)}=\mu^{p-1}\left(1+\left(\mu^{p-1}-\dot{\phi}\right)+\left(\mu^{p-1}-\dot{\phi}\right)^{2}+\ldots\right) \text {. }
$$

Notice, that in the product on the right, any terms involving $\phi_{1}, \phi_{2}, \ldots$, or $\phi_{p-1}$ ultimately yield zero when multiplied by $\mu^{p-1}$. Hence $\dot{\phi}$ may be replaced 
by $\phi_{p}$, and we obtain, as required,

$$
\frac{\mu^{p-1}}{1-\left(\mu^{p-1}-\phi_{p}\right)}=\mu^{p-1}\left(1+\mu^{p-1}-\phi_{p}+\left(\mu^{p-1}-\phi_{p}\right)^{2}+\ldots\right) \text {. }
$$

Let $N(k)=\Pi p^{[k /(p-1)]} / k$ ! as before, and put $\delta_{k}=s_{k}-p_{k}$.

THEOREM II. With the notation as above, in $P \int T, p \delta_{k}=0$ in any event; and $\delta_{k} \neq 0 \Leftrightarrow p \mid N(k)$.

Note. In essence, Theorem II tells us the exact order of the difference $s_{k}$ (ind $\left.\xi\right)-\operatorname{tr}\left(s_{k}(\xi)\right)$ for $\xi: T^{p} \rightarrow T=U_{1}$ the projection onto the first factor. It turns out to be the power $(<1)$ of $p$ in $\bar{N}(k)$.

Proof of Theorem II. For $k$ a natural number, set $k=p^{p_{p}(k)} k^{\prime}$ where $\left(k^{\prime}, p\right)=1$. Then one knows that

$$
\nu_{p}(k !)=\left[\frac{k}{p}\right]+\left[\frac{k}{p^{2}}\right]+\cdots+\left[\frac{k}{p^{d}}\right]+\ldots
$$

Hence,

$$
\nu_{p}(N(k))=\left[\frac{k}{p-1}\right]-\left[\frac{k}{p}\right]-\left[\frac{k}{p^{2}}\right]-\cdots-\left[\frac{k}{p^{d}}\right]-\ldots
$$

Write

$$
\begin{aligned}
& k=k_{1} p+r_{0} \\
& k_{1}=k_{2} p+r_{1} \\
& \vdots \\
& k_{d}=k_{d+1} p+r_{d}
\end{aligned}
$$

where $0<r_{j}<p, j=0,1,2, \ldots$, and note

$$
k=r_{0}+r_{1} p+r_{2} p^{2}+\cdots+r_{d} p^{d}+\ldots
$$

is the $p$-adic expansion of $k$. Also, $k_{1}=[k / p], k_{2}=\left[k_{1} / p\right]=\left[k / p^{2}\right]$, etc.

We can rewrite

$$
\begin{aligned}
& k=k_{1}(p-1)+k_{1}+r_{0} \\
& k_{1}=k_{2}(p-1)+k_{2}+r_{1}
\end{aligned}
$$

Hence,

$$
\left[\frac{k}{p-1}\right]=k_{1}+k_{2}+\cdots+k_{d}+\cdots+\left[\frac{r_{0}+r_{1}+r_{2}+\ldots}{p-1}\right] .
$$


Thus,

$$
\nu_{p}(N(k))=\left[\frac{r_{0}+r_{1}+\cdots+r_{d}+\ldots}{p-1}\right]
$$

and

$$
\nu_{p}(N(k))>0 \Leftrightarrow r_{0}+r_{1}+\cdots+r_{d}+\ldots>p-1 .
$$

Our task now is to determine the nonzero terms in the expansion of Proposition I,

$$
\sum_{k=1}^{\infty}(-1)^{k+1} \delta_{k}=\sum_{n=0}^{\infty} \mu^{p-1}\left(\mu^{p-1}-\phi_{p}\right)^{n} .
$$

(In any event, $p \mu=0$ so $p \delta_{k}=0$ for all $k>1$.)

Fix an $n$ in (F2), and write

$$
n=s_{0}+s_{1} p+s_{2} p^{2}+\cdots+s_{d} p^{d}+\ldots
$$

with $0<s_{j}<p, j=0,1,2, \ldots$

Then

$$
\begin{aligned}
\left(\mu^{p-1}-\phi_{p}\right)^{n} \equiv\left(\mu^{p-1}-\phi_{p}\right)^{s_{0}}\left(\mu^{(p-1) p}-\phi_{p}^{p}\right)^{s_{1}} & \ldots \\
& \left(\mu^{(p-1) p^{d}}-\phi_{p}^{p^{d}}\right)^{s_{d}} \ldots
\end{aligned}
$$

The congruence is modulo $p$, but after multiplication by $\mu^{p-1}$, it may be replaced by equality. Suppose

$$
i=t_{0}+t_{1} p+t_{2} p^{2}+\cdots+t_{d} p^{d}+\ldots
$$

with $0<t_{j}<s_{j}, j=0,1,2, \ldots$ We shall abbreviate these coefficient inequalities by $i<_{p} n$. Then, for each $i<_{p} n$, the term

$$
\pm h_{n, i} \mu^{i(p-1)} \phi_{p}^{n-i}
$$

occurs on the right where

$$
h_{n, i}=\left(\begin{array}{c}
s_{0} \\
t_{0}
\end{array}\right)\left(\begin{array}{c}
s_{1} \\
t_{1}
\end{array}\right) \ldots\left(\begin{array}{c}
s_{d} \\
t_{d}
\end{array}\right) \ldots \neq 0 \bmod p .
$$

Moreover, these are the only terms which occur there. Thus, the expression on the right of (F2) is a sum of nonzero terms of the form

$$
\pm h_{n, i} \mu^{p-1} \mu^{i(p-1)} \phi_{p}^{n-i} \quad \text { with } i<_{p} n \text {. }
$$

Looking at the degrees in $\mu$ and $\phi_{p}$ separately, we see that these terms form a linearly independent set. Moreover, the total degree (divided by 2) in (F4) is $p-1+i(p-1)+(n-i) p=p-1+n p-i$. Thus,

$$
\delta_{k} \neq 0 \Leftrightarrow \mid \begin{aligned}
& \text { There is a representation } \\
& k=p-1+n p-i \\
& \text { with } n, i>0 \text { and } i<_{p} n .
\end{aligned}
$$


Thus, we need to show the equivalence of the conditions on the right of (L1) and (L2).

LEMMA 3. With the notation as above, if $k=p-1+n p-i$ with $n, i>0$ and $i<_{p} n$, then $r_{0}+r_{1}+\cdots+r_{d}+\ldots>p-1$.

Proof.

$$
\begin{aligned}
k= & p-1+\left(s_{0}+s_{1} p+\ldots\right) p-t_{0}-t_{1} p-\ldots \\
= & \left(p-1-t_{0}\right)+\left(\varepsilon_{1} p+s_{0}-t_{1}\right) p+\left(\varepsilon_{2} p+s_{1}-t_{2}-\varepsilon_{1}\right) p^{2} \\
& +\cdots+\left(\varepsilon_{d} p+s_{d-1}-t_{d}-\varepsilon_{d-1}\right) p^{d}+\ldots
\end{aligned}
$$

where $\varepsilon_{0}=0$ and inductively

$$
\varepsilon_{d}= \begin{cases}0 & \text { if } s_{d-1}-t_{d}-\varepsilon_{d-1}>0 \\ 1 & \text { if } s_{d-1}-t_{d}-\varepsilon_{d-1}<0\end{cases}
$$

Note, in any event, $-p<s_{d-1}-t_{d}-\varepsilon_{d-1}<p-1$, so that

$$
0<\varepsilon_{d} p+s_{d-1}-t_{d}-\varepsilon_{d-1}<p \text {. }
$$

Thus, we have determined the $p$-adic expansion of $k$.

$$
\begin{aligned}
& r_{0}=p-1-t_{0} \\
& r_{1}=\varepsilon_{1} p+s_{0}-t_{1} \\
& r_{2}=\varepsilon_{2} p+s_{1}-t_{2}-\varepsilon_{1} \\
& \vdots \\
& r_{d}=\varepsilon_{d} p+s_{d-1}-t_{d}-\varepsilon_{d-1}
\end{aligned}
$$

Hence,

$$
\begin{array}{r}
r_{0}+r_{1}+\cdots+r_{d}+\ldots=p-1+\left(\varepsilon_{1}+\varepsilon_{2}+\cdots+\varepsilon_{d}+\ldots\right)(p-1) \\
+s_{0}-t_{0}+s_{1}-t_{1}+\cdots+s_{d}-t_{d}+\ldots
\end{array}
$$

However,

$$
\varepsilon_{1}+\varepsilon_{2}+\cdots+\varepsilon_{d}+\ldots \geqslant 0
$$

and, since $i<_{p} n$, by definition,

$$
s_{0}-t_{0}+s_{1}-t_{1}+\cdots+s_{d}-t_{d}+\ldots>0 .
$$

Thus, $r_{0}+r_{1}+\ldots>p-1$ as required.

LEMMA 4. With the notation as above, if $r_{0}+r_{1}+\ldots>p-1$, then $k=p-1+n p-i$ with $n, i>0$ and $i<_{p} n$. 
Proof.

$$
\begin{aligned}
k & =r_{0}+r_{1} p+r_{2} p^{2}+\ldots \\
& =p-1+\left(r_{1}+r_{2} p+\ldots\right) p-\left(p-1-r_{0}\right) .
\end{aligned}
$$

Try $i=t_{0}=p-1-r_{0}, n=r_{1}+r_{2} p+\ldots=k_{1}$. If $i \leqslant_{p} n$, we are done. But,

$$
t_{0} \leqslant r_{1} \Leftrightarrow p-1-r_{0} \leqslant r_{1} \Leftrightarrow p-1 \leqslant r_{0}+r_{1} .
$$

If $t_{0}>r_{1}$, rewrite

$$
\begin{aligned}
k & =p-1+\left(t_{0}+r_{2} p+\ldots\right) p-\left(t_{0}+\left(t_{0}-r_{1}\right) p\right) \\
& =p-1+\left(t_{0}+r_{2} p+\ldots\right) p-\left(t_{0}+t_{1} p\right)
\end{aligned}
$$

where $0<t_{1}=t_{0}-r_{1}=p-1-r_{0}-r_{1}(<p)$. Try $i=t_{0}+t_{1} p, n=t_{0}+$ $r_{2} p+\ldots$ Then

$$
i \leqslant p n \Leftrightarrow t_{1} \leqslant r_{2} \Leftrightarrow p-1-r_{0}-r_{1} \leqslant r_{2} \Leftrightarrow p-1 \leqslant r_{0}+r_{1}+r_{2} .
$$

Continue in this way. Let $d$ be the first index such that

$$
r_{0}+r_{1}+r_{2}+\cdots+r_{d}>p-1 \text {. }
$$

Define

$$
t_{j}=t_{j-1}-r_{j}=p-1-r_{0}-r_{1}-\cdots-r_{j}>0
$$

for $j=0,1, \ldots, d-1$. Then

$$
\begin{aligned}
k= & p-1+\left(t_{0}+t_{1} p+\cdots+r_{d} p^{d-1}+\ldots\right) p \\
& -\left(t_{0}+t_{1} p+\cdots+t_{d-1} p^{d-1}\right),
\end{aligned}
$$

and we can take

$$
\begin{aligned}
i & =t_{0}+t_{1} p+\cdots+t_{d-1} p^{d-1}, \\
n & =t_{0}+t_{1} p+\cdots+r_{d} p^{d-1}+\ldots
\end{aligned}
$$

Since $t_{d-1}=p-1-r_{0}-r_{1}-\cdots-r_{d-1} \leqslant r_{d}$, we are done.

3. The general case. We shall show that $\bar{N}(k)=\Pi_{p \mid N(k)} p$ is the optimal bound. First, we exhibit a class of groups for which $\bar{N}(k)$ is necessary.

For $p$ a prime, let $\lambda_{p}: P \rightarrow T$ be a nontrivial 1-dimensional representation, and consider the $p$-dimensional representation of $G_{p}=P \int P$ given by $\rho_{p}=$ $\iota^{\prime} \circ\left(\right.$ id $\left.\int \lambda_{p}\right)$ :

$$
P \int P \stackrel{\mathrm{id} \int \lambda_{p}}{\rightarrow} P \int T \stackrel{\iota^{\prime}}{\rightarrow} U_{p} .
$$

Morever, let $\xi_{p}=\imath \circ\left(\lambda_{p} \times 1 \times \cdots \times 1\right)$ :

$$
H_{p}=P^{p} \stackrel{\lambda_{p} \times 1 \times \cdots \times 1}{\longrightarrow} T^{p} \rightarrow U_{p} .
$$

Then $\rho_{p}=$ ind $\xi_{p}$. Note $s_{k}\left(\xi_{p}\right)=s_{k}\left(\lambda_{p}\right) \times 1 \times \cdots \times 1$ where $\lambda_{p}$ is viewed in $H^{2}(P, \mathbf{Z}) \cong \operatorname{Hom}(P, T)$. Also, $s_{k}\left(\lambda_{p}\right)=\lambda_{p}{ }^{k}$. 
We have a commutative diagram

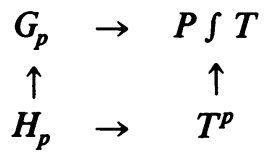

which yields a transfer diagram

$$
\begin{array}{ccc}
H^{2 k}\left(G_{p}, \mathbf{Z}\right) & \leftarrow & H^{2 k}\left(B\left(P \int T\right), \mathbf{Z}\right) \\
\uparrow \operatorname{Tr} & & \uparrow \operatorname{Tr} \\
H^{2 k}\left(H_{p}, \mathbf{Z}\right) & \leftarrow & H^{2 k}\left(B\left(T^{p}\right), \mathbf{Z}\right)
\end{array}
$$

On the other hand, a simple calculation shows

$$
\begin{aligned}
p_{k} & =x_{1}^{k}+\cdots+x_{p}^{k} \\
& =\operatorname{Tr}_{T^{p} \rightarrow P \int T}\left(x_{1}^{k}\right) \in H^{0}\left(P, H^{2 k}\left(B T^{p}\right)\right) \subset H^{2 k}\left(B\left(P \int T\right)\right),
\end{aligned}
$$

where $x_{1}^{k} \in H^{2 k}\left(B T^{p}, \mathbf{Z}\right)=$ component of $\mathbf{Z}\left[x_{1}, \ldots, x_{p}\right]$ of degree $k$. (See [Ch1, §2, pp. 181-182]. We make use of the Nakaoka isomorphism and its naturality with respect to transfer.)

Hence,

$$
\begin{aligned}
s_{k}\left(\rho_{p}\right) & =\left(\operatorname{id} \int \lambda_{p}\right)^{*}\left(s_{k}\right)=\left(\operatorname{id} \int \lambda_{p}\right)^{*}\left(p_{k}+\delta_{k}\right) \\
& =\left(\operatorname{id} \int \lambda_{p}\right)^{*}\left(\operatorname{Tr} x_{1}^{k}\right)+\left(\operatorname{id} \int \lambda_{p}\right)^{*}\left(\delta_{k}\right) \\
& =\operatorname{Tr}\left(\lambda_{p} \times 1 \times \cdots \times 1\right)^{*}\left(x_{1}^{k}\right)+\Delta_{k p} \\
& =\operatorname{Tr}\left(\lambda_{p}^{*}\left(x_{1}\right)^{k} \times 1 \times \cdots \times 1\right)+\Delta_{k p}
\end{aligned}
$$

where $\Delta_{k p}=\left(\text { id } \int \lambda_{p}\right)^{*}\left(\delta_{k}\right)$. Thus,

$$
s_{k}\left(\rho_{p}\right)-\operatorname{Tr}\left(s_{k}\left(\xi_{p}\right)\right)=\Delta_{k, p}
$$

We shall show

$$
\Delta_{k p} \neq 0 \Leftrightarrow \delta_{k} \neq 0 \Leftrightarrow p \mid N(k) .
$$

To see this, note first that $\delta_{k} \in H^{*}\left(B\left(P \int T\right), \mathbf{Z}\right)$. Also, if $\bar{\mu} \in$ $H^{2}(P, \mathrm{Z} / p \mathrm{Z})$ is the reduction $\bmod p$ of $\mu$, then the homomorphism

$$
\mu H^{*}\left(B\left(P \int T\right), Z\right) \rightarrow \bar{\mu} H^{*}\left(B\left(P \int T\right), Z / p Z\right)
$$

is a monomorphism. (For, we may use the Nakaoka isomorphisms and note that $H^{2 r}(P, \mathbf{Z}) \rightarrow H^{2 r}(P, \mathbf{Z} / p \mathbf{Z}), \quad r>0$, is an isomorphism. Since $H^{r}\left(P, Z\left[x_{1}, \ldots, x_{p}\right]\right) \simeq \bigoplus H^{r}(P, Z)$ for $r>0$ by the $P$-module argument of $\$ 2$, the connection is clear.) Moreover, for coefficients in $\mathrm{Z} / p \mathrm{Z}$, the Nakaoka argument works also for $P \int P$, and we have a commutative diagram

$$
\begin{gathered}
H^{*}\left(P \int P, \mathrm{Z} / p \mathrm{Z}\right) \leftarrow H^{*}(B(P \leq \underset{\mathbb{R}}{T}), \mathrm{Z} / p \mathrm{Z}) \\
H^{*}\left(P, \mathrm{Z} / p \mathrm{Z}\left[u_{1}, u_{2}, \ldots, u_{p}, \beta u_{1}, \ldots, \beta u_{p}\right]\right) \leftarrow H^{*}\left(P, \mathrm{Z} / p \mathrm{Z}\left[\bar{x}_{1}, \ldots, \bar{x}_{p}\right]\right)
\end{gathered}
$$


where $u_{1}, \ldots, u_{p}$ form a basis for $H^{1}\left(P^{p}, \mathrm{Z} / p \mathrm{Z}\right)$ generating an exterior algebra, and their Bocksteins $\beta u_{1}, \ldots, \beta u_{p}$ generate a polynomial subring. The lower arrow is induced from $\bar{x}_{i} \rightarrow \beta u_{i}, i=1,2, \ldots, p$.

Since $\mathbf{Z} / p \mathbf{Z}\left[\bar{x}_{1}, \ldots, \bar{x}_{p}\right] \cong \mathbf{Z} / p \mathbf{Z}\left[\beta u_{1}, \ldots, \beta u_{p}\right]$ is a $P$-direct summand of $H^{*}\left(P^{p}, \mathrm{Z} / p \mathrm{Z}\right)$, it follows that the lower (and hence upper) arrow is a monomorphism. That $\Delta_{k, p} \neq 0 \Leftrightarrow \delta_{k} \neq 0$ follows from the commutative diagram:

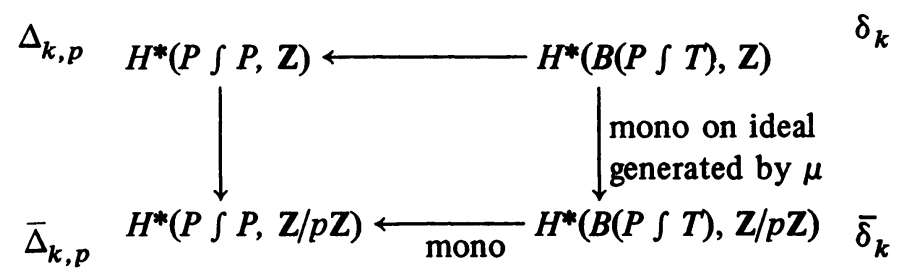

Note. If we write $\omega_{p}=\left(\mathrm{id} \int \lambda_{p}\right)^{*}\left(\phi_{p}\right)$, then we have the formula

$$
\sum_{k=1}^{\infty}(-1)^{k+1} \Delta_{k p}=\frac{\mu^{p-1}}{1-\mu^{p-1}+\omega_{p}} .
$$

( $\omega_{p}$ is just the wreath product class: $\omega_{p}=1 \int \lambda_{p}$. See [Ch1, Theorem 3] and [N, §4].) Similarly, if we take $\lambda_{p}=1$ instead, then id $\int 1=\pi_{p}, \operatorname{Tr}\left(s_{k}(1)\right)=0$ for $k>0$, and we obtain the formula

$$
\sum_{k=1}^{\infty}(-1)^{k+1} s_{k}\left(\pi_{p}\right)=\frac{\mu^{p-1}}{1-\mu^{p-1}} .
$$

Thus,

$$
s_{k}\left(\pi_{p}\right)= \begin{cases}0, & k \neq 0 \bmod p-1, \\ \pm \mu^{k}, & k \equiv 0 \bmod p-1 .\end{cases}
$$

We shall use these facts later.

On the basis of (I1), we can conclude that the required bound is no smaller than $\bar{N}(k)$. For each prime $p$ dividing $\bar{N}(k)$, there is a group $P \int P$ and induced representation $\rho_{p}$ such that $s_{k}\left(\rho_{p}\right)$ is of order $p$. (However, one can construct a single group $\bar{G}_{k}$, and an induced representation $\rho_{k}$ for which $s_{k}\left(\bar{\rho}_{k}\right)$ is of order $\bar{N}(k)$ so that all primes are dealt with simultaneously by the same group. To do so requires an extended discussion which is not essential for our basic argument.)

We now show that $\bar{N}(k)$ is a universal bound for all groups.

THEOREM III. Fix an integer $k>0$, and let $\bar{N}(k)$ be the product of all distinct primes $p$ which divide $N(k)=\left(\Pi_{p} p^{[k /(p-1)]}\right) / k$ !. Then for every finite 
group $G$, subgroup $H$, and finite dimensional unitary representation $\xi$ of $H$,

$$
\bar{N}(k)\left[s_{k}\left(\operatorname{ind}_{H \rightarrow G} \xi\right)-\operatorname{Tr}_{H \rightarrow G}\left(s_{k}(\xi)\right)\right]=0 .
$$

Moreover, $\bar{N}(k)$ is the least natural number with this property.

Proof. We have just dealt with the second assertion. For the first assertion, we proceed as follows.

First, we reduce to the case of $p$-groups. To do so, consider general $G \geqslant H$ and $\xi$. For each prime $p$, let $Q$ be a $p$-Sylow subgroup of $G$. For any $s \in H^{*}(H, \mathrm{Z})$, we have the double coset rule [CE, Chapter XII, Proposition 9.1].

$$
\operatorname{res}_{G \rightarrow Q}\left(\operatorname{tr}_{H \rightarrow G}(s)\right)=\sum_{i=1}^{d} \operatorname{tr}_{H_{i} \cap Q \rightarrow Q}\left(\operatorname{res}_{H_{i} \rightarrow H_{i} \cap Q}\left(c_{i}(s)\right)\right)
$$

where $G=\cup_{i=1}^{d} Q g_{i} H$ is a double coset decomposition of $G, H_{i}=g_{i} H g_{i}^{-1}$, and $c_{i}: H^{*}(H, Z) \rightarrow H^{*}\left(H_{i}, \mathrm{Z}\right)$ is induced by the homomorphism $H_{i} \rightarrow H$ given by $h^{\prime} \rightarrow g_{i}^{-1} h^{\prime} g_{i}$. Similarly, for $\xi$ a representation of $H$, the original double coset rule for induced representations reads

$$
\operatorname{res}_{G \rightarrow Q}\left(\operatorname{ind}_{H \rightarrow G} \xi\right)=\sum_{i=1}^{d} \operatorname{ind}_{H_{i} \cap Q \rightarrow Q}\left(\operatorname{res}_{H_{i} \rightarrow H_{i} \cap Q} c_{i}(\xi)\right)
$$

(as above, $\left.c_{i}(\xi)\left(h^{\prime}\right)=\xi\left(g_{i}^{-1} h^{\prime} g_{i}\right)\right)$.

Since restriction from $G$ to $Q$ is a monomorphism on the p-primary component, to prove the theorem, it suffices to prove (for $k>0$ )

$$
\bar{N}(k)\left[\operatorname{res}_{G \rightarrow Q}\left(s_{k}\left(\operatorname{ind}_{H \rightarrow G} \xi\right)\right)-\operatorname{Tr}_{H \rightarrow G}\left(s_{k}(\xi)\right)\right]=0
$$

for every prime $p$ dividing the order of $G$. Using the two double coset rules (and the properties (a) and (b) of $s_{k}$ ), we can reduce the expression in brackets in $(\mathrm{P})$ to

$$
\sum_{i=1}^{d}\left[s_{k}\left(\operatorname{ind}_{H_{i} \cap Q \rightarrow Q} \xi_{i}\right)-\operatorname{Tr}_{H_{i} \cap Q \rightarrow Q}\left(s_{k}\left(\xi_{i}\right)\right)\right]
$$

where we abbreviate $\xi_{i}=\operatorname{res}_{H_{i} \rightarrow H_{i} \cap Q}\left(c_{i}(\xi)\right)$. If we establish the theorem for $p$-groups, then $\bar{N}(k)$ works for each term of the sum and hence for the sum.

We now suppose that $G$ is a $p$-group. Because of transitivity, we may further assume that $H$ is a (necessarily normal) subgroup of index $p$. For, let $G>G_{1}>H$, and suppose the theorem has been established for index $<(G$ : 
$H)$. Then,

$$
\begin{aligned}
\bar{N}(k)\left[s_{k}\left(\operatorname{ind}_{H \rightarrow G} \xi\right)-\right. & \left.\operatorname{Tr}_{H \rightarrow G}\left(s_{k}(\xi)\right)\right] \\
=\bar{N}(k)[ & s_{k}\left(\operatorname{ind}_{G_{1} \rightarrow G}\left(\operatorname{ind}_{H \rightarrow G} \xi\right)\right)-\operatorname{Tr}_{G_{1} \rightarrow G}\left(s_{k}\left(\operatorname{ind}_{H \rightarrow G} \xi\right)\right) \\
& \left.\quad \operatorname{Tr}_{G_{1} \rightarrow G}\left(s_{k}\left(\operatorname{ind}_{H \rightarrow G} \xi\right)\right)-\operatorname{Tr}_{G_{1} \rightarrow G}\left(\operatorname{Tr}_{H \rightarrow G_{1}}\left(s_{k}(\xi)\right)\right)\right] \\
=\bar{N}(k)[ & \left.s_{k}\left(\operatorname{ind}_{G_{1} \rightarrow G} \xi_{1}\right)-\operatorname{Tr}_{G_{1} \rightarrow G} s_{k}\left(\xi_{1}\right)\right] \\
& \quad \operatorname{Tr}_{G_{1} \rightarrow G}\left(\bar{N}(k)\left[s_{k}\left(\operatorname{ind}_{H \rightarrow G} \xi\right)-\operatorname{Tr}_{H \rightarrow G_{1}}\left(s_{k}(\xi)\right)\right]\right) .
\end{aligned}
$$

(We have abbreviated $\xi_{1}=\operatorname{ind}_{H \rightarrow G} \xi$.) However, both terms in this expression have been inductively assumed to be zero.

We can now suppose that $H$ is a normal subgroup of $G$ of index $p$. If so, there is a factorization of the induced representation, $\rho=\operatorname{ind}_{H \rightarrow G} \xi$,

$$
G \stackrel{\Phi}{\rightarrow} P \int H \stackrel{\text { id } \xi}{\rightarrow} P \int U_{n} \stackrel{i}{\rightarrow} U_{p n}
$$

where $n=\operatorname{deg} \xi$. ( $\Phi$ is defined in [N, §2, pp. 54-55]; see also [Ch1, p. 189] and [Ch2].) Hence, we must analyze the class $s_{k}(\iota) \in H^{2 k}\left(B\left(P \int U_{n}\right), Z\right)$. To this end, recall the Nakaoka isomorphism (\$2)

$$
H^{*}\left(B\left(P \int U_{n}\right), \mathrm{Z}\right) \cong H^{*}\left(P, H^{*}\left(B U_{n}, \mathrm{Z}\right)^{p}\right) .
$$

Let $S_{k}$ be the universal class in $H^{2 k}\left(B U_{n}, \mathrm{Z}\right)$, and write

Then

$$
\begin{gathered}
s_{k, i}=1 \times 1 \times \cdots \times S_{\uparrow} \times \cdots \times 1 . \\
i \text { th position }
\end{gathered}
$$

$$
\operatorname{tr}\left(s_{k, 1}\right)=s_{k, 1}+s_{k, 2}+\cdots+s_{k, p} \in H^{2 k}\left(\left(B U_{n}\right)^{p}, \mathbf{Z}\right)^{P}
$$

LEMMA. With the notation as above, for $k>0$,

$$
\bar{N}(k)\left[s_{k}(\bar{\imath})-\operatorname{tr}\left(s_{k, 1}\right)\right]=0 .
$$

Proof of Lemma. One knows that

$$
H^{*}\left(B U_{n}, \mathbf{Z}\right) \rightarrow H^{*}\left(B T^{n}, \mathbf{Z}\right)=\mathbf{Z}\left[x_{1}, x_{2}, \ldots, x_{n}\right]
$$

is a monomorphism onto the direct summand of symmetric polynomials. (See [Bor, §20, p. 66].) Hence,

$$
H^{*}\left(B U_{n}, \mathrm{Z}\right)^{p} \rightarrow H^{*}\left(B T^{n}, \mathrm{Z}\right)^{p}
$$

is $P$-monomorphism onto a $P$-direct summand. Hence, the restriction

$$
\begin{aligned}
H^{*}\left(B\left(P \int U_{n}\right)\right) & \cong H^{*}\left(P, H^{*}\left(B U_{n}\right)^{p}\right) \\
& \rightarrow H^{*}\left(P, H^{*}\left(B T^{n}\right)^{p}\right) \cong H^{*}\left(B\left(P \int T^{n}\right)\right)
\end{aligned}
$$


is also a monomorphism, and it suffices to prove an analogous result on the right. In more detail, let $P^{*} \cong P$ be the subgroup of $\delta_{n p}$ which permutes the $p$ blocks $\{1,2, \ldots, n\},\{n+1, \ldots, 2 n\}, \ldots,\{(p-1) n+1, \ldots, p n\}$ cyclically. Then,

$$
P \int_{p}\left(T^{n}\right) \cong P \int_{\rho}\left(1 \int_{n} T\right) \cong\left(P \int_{p} 1\right) \int_{p n} T=P^{*} \int_{p n} T .
$$

Writing

$$
\begin{aligned}
& H^{*}\left(B T^{p n}\right)=H^{*}\left(B T^{n}\right)^{p} \\
& \cong \mathbf{Z}\left[x_{11}, \ldots, x_{1 n}\right] \otimes \mathbf{Z}\left[x_{21}, \ldots, x_{2 n}\right] \otimes \cdots \otimes \mathbf{Z}\left[x_{p 1}, \ldots, x_{p n}\right] \\
& \simeq \mathbf{Z}\left[\left\{x_{i j} \mid i=1, \ldots, p ; j=1, \ldots, n\right\}\right], \\
& p_{k, i}=x_{i 1}^{k}+x_{i 2}^{k}+\cdots+x_{i n}^{k}, \text { we note that } s_{k, i} \rightarrow p_{k, i} \text {, and } \\
& \qquad \operatorname{tr}\left(p_{k, 1}\right)=p_{k, 1}+p_{k, 2}+\cdots+p_{k, p}=\sum_{i, j} x_{i j}^{k} .
\end{aligned}
$$

(Denote this last sum simply $p_{k}^{\prime}$.) Thus, if $\iota^{\prime}: P^{*} \int_{p n} T \rightarrow U_{p n}$ is the inclusion, we need to show

$$
\bar{N}(k)\left[s_{k}\left(\iota^{\prime}\right)-p_{k}^{\prime}\right]=0 .
$$

(Transfer commutes with restriction in this case since

$$
\left.\left(P \int_{p} U_{n}: U_{n}^{p}\right)=\left(P \int_{p} T^{n}:\left(T^{n}\right)^{p}\right)={ }_{1} p .\right)
$$

To establish (K1), it suffices to work in any larger wreath product $S \int_{p n} T$ where $P<S<\delta_{n p}$. If we do so, $p_{k}^{\prime}=\Sigma_{i, j} x_{i j}^{k} \in H^{2 k}\left(B T^{n p}, \mathrm{Z}\right)^{S}$ need not be changed, but $\iota^{\prime}$ is replaced by the inclusion $\iota: S \int_{p n} T \rightarrow U_{p n}$.

The appropriate group to use is $S=P^{n}$ where the $j$ th factor $P$ of the product permutes the $j$ th column $\left\{x_{i j} \mid i=1,2, \ldots, p\right\}$ of the matrix of indeterminates. $P^{*}$ is imbedded diagonally in $P^{n}$. For this choice,

$$
P^{n} \int_{p n} T \cong\left(P \int_{p} T\right)^{n}
$$

and

$$
\iota^{\prime \prime} \cong \iota \times \iota \times \cdots \times \iota \quad(n \text { times })
$$

where $\iota: P \int \boldsymbol{T} \rightarrow U_{p}$ is the inclusion studied in $\$ 2$. Moreover,

$$
\begin{aligned}
s_{k}(\iota \times \iota \times \cdots \times \iota)= & s_{k}(\iota) \times 1 \times \cdots \times 1+1 \times s_{k}(\iota) \times \cdots \times 1 \\
& +\cdots+1 \times 1 \times \cdots \times s_{k}(\iota) .
\end{aligned}
$$

On the other hand, $p_{k}^{\prime}$ may be rewritten

$$
\begin{aligned}
p_{k}^{\prime}= & \sum_{j=1}^{n} \sum_{i=1}^{p} x_{i j}^{k}=p_{k} \times 1 \times \cdots \times 1+1 \times p_{k} \times \cdots \times 1 \\
& +\ldots+1 \times 1 \times \cdots \times p_{k}
\end{aligned}
$$


where (as in §2) $p_{k}=x_{11}^{k}+x_{21}^{k}+\cdots+x_{p 1}^{k}$, and we make suitable identifications involving cross products. Subtract (K3) from (K2) and recall from Theorem II that $\bar{N}(k)\left[s_{k}(\iota)-p_{k}\right]=0$. That completes the proof of the lemma.

Note. Subtler versions of the above arguments are used in [Ch2] to calculate the Chern classes of $\iota$.

To prove the theorem, consider the diagram

$$
\begin{array}{ccccc}
G & \stackrel{\Phi}{\rightarrow} & P \int H & \stackrel{\text { idf } \xi}{\rightarrow} & P \int U_{n} \\
\uparrow & & \uparrow & & \uparrow \\
H & \stackrel{\Phi^{\prime}}{\rightarrow} & H^{p} & \stackrel{\xi^{p}}{\rightarrow} & U_{n}^{p}
\end{array}
$$

where $\Phi^{\prime}$ is the restriction of $\Phi$ to the normal subgroup $H$. In fact,

$$
\Phi^{\prime}(h)=h \times g_{2}^{-1} h g_{2} \times \cdots \times g_{p}^{-1} h g_{p}
$$

where $g_{1}=1, g_{2}, \ldots, g_{p}$ forms a set of left coset representatives of $H$ in $G$. (See [N, §2].) The subgroups in the diagram (K4) are each of index $p$, and there results a commutative transfer diagram. Thus,

$$
\begin{aligned}
\left(\operatorname{id} \int \xi\right)^{*}\left(\operatorname{tr}\left(s_{k, 1}\right)\right) & =\operatorname{tr}\left(\left(\xi^{p}\right)^{*}\left(s_{k, 1}\right)\right) \\
& =\operatorname{tr}\left((\xi \times \cdots \times \xi)^{*}\left(S_{k} \times 1 \times \cdots \times 1\right)\right) \\
& =\operatorname{tr}\left(\xi^{*}\left(S_{k}\right) \times 1 \times \cdots \times 1\right) \\
& =\operatorname{tr}\left(s_{k}(\xi) \times 1 \times \cdots \times 1\right) .
\end{aligned}
$$

Hence

$$
\begin{aligned}
\Phi^{*}\left(\left(\operatorname{id} \int \xi\right)^{*}\left(\operatorname{tr}\left(s_{k, 1}\right)\right)\right) & =\Phi^{*}\left(\operatorname{tr}\left(s_{k}(\xi) \times \cdots \times 1\right)\right) \\
& =\operatorname{tr}\left(\Phi^{\prime *}\left(s_{k}(\xi) \times 1 \times \cdots \times 1\right)\right) \\
& =\operatorname{tr}\left(s_{k}(\xi)\right) .
\end{aligned}
$$

Since $\Phi^{*}\left(\left(\operatorname{id} \int \xi\right)^{*}\left(s_{k}(\emptyset)\right)\right)=s_{k}(\rho)$, we have

$$
s_{k}(\rho)-\operatorname{tr}\left(s_{k}(\xi)\right)=\Phi^{*}\left(\left(\operatorname{id} \int \xi\right)^{*}\left[s_{k}(\bar{\imath})-\operatorname{tr}\left(s_{k, 1}\right)\right]\right) .
$$

The theorem now follows from the lemma.

4. Some examples. We show in several examples that the classes $s_{k}(\rho)$ contain less information, in some sense, than the Chern classes.

(a) The regular representation. Let $G$ be a finite group and $\rho_{G}$ its regular representation. Then, $\rho=\operatorname{ind}_{\{1\} \rightarrow G} 1$, and since $\operatorname{Tr}\left(s_{k}(1)\right)=\operatorname{Tr}(0)=0$ for $k>0$, we conclude from Theorem III

$$
\bar{N}(k) s_{k}\left(\rho_{G}\right)=0, \quad k>0 .
$$

In particular if $G$ is a $p$-group, then $p s_{k}\left(\rho_{G}\right)=0$ for $k>0$. 
One knows, on the other hand, from an argument of Venkov [Q, §2, Theorem 2.1], that $H^{*}(G, \mathrm{Z})$ is a finite module over the subring $\mathbf{Z}\left[c_{1}\left(\rho_{G}\right), c_{2}\left(\rho_{G}\right), \ldots, c_{g}\left(\rho_{G}\right)\right]$ generated by the Chern classes. It follows that the exponent of $H^{r}(G, \mathrm{Z})$, for $r$ sufficiently large, cannot be larger than the largest order of any of the Chern classes. Hence, the Chern classes of the regular representation generally have large orders while the orders of the classes $s_{k}\left(\rho_{G}\right)$ are small.

Note. To emphasize this point more concretely, we give the answers for a cyclic group $G$ of order $p^{m}$ (without proofs). $\chi$ denotes a generator of $H^{2}(G, \mathbf{Z})$.

(i) $p$ odd,

$$
c\left(\rho_{G}\right)=\left(1-\chi^{p-1}\right)^{p^{m-1}}
$$

hence,

$$
c_{p^{m}-p^{m-1}}\left(\rho_{G}\right)=-\chi^{p^{m}-p^{m-1}}
$$

but

$$
s_{k}\left(\rho_{G}\right)= \begin{cases}p^{m-1} \chi^{k}, & k \equiv 0 \bmod p-1 \\ 0, & \text { otherwise. }\end{cases}
$$

(ii) $p=2$;

$$
c\left(\rho_{G}\right)=[(1+\chi)(1+2 \chi)(1+3 \chi)]^{2^{m-2}},
$$

but

$$
s_{k}\left(\rho_{G}\right)= \begin{cases}2^{m-1} \chi^{k}, & k \text { even } \\ 0, & k \text { odd }\end{cases}
$$

(b) Cyclic groups.

Proposition IV. Let $G$ be cyclic of order $p^{m}, m \geqslant 2, H$ a subgroup of index $p, \lambda \in H^{2}(G, Z)$, and $\xi=\operatorname{res}_{G \rightarrow H} \lambda$. Then, for $p$ odd,

$$
s_{k}(\text { ind } \xi)=p \lambda^{k}=\operatorname{tr}\left(s_{k}(\xi)\right) \text {, }
$$

and for $p=2$,

$$
\begin{aligned}
s_{k}(\text { ind } \xi) & =2 \lambda^{k}=\operatorname{tr}\left(s_{k}(\xi)\right), \quad k \text { even, } \\
& =2 \lambda^{k}+2^{m-1} \lambda^{k}=\operatorname{tr}\left(s_{k}(\xi)\right)+2^{m-1} \lambda^{k}, \quad k \text { odd }
\end{aligned}
$$

Proof. Use Frobenius reciprocity and calculate.

Corollary V. Let $G$ be cyclic or order $p^{m}, m \geqslant 2, H$ any nontrivial subgroup, and $\xi$ a representation of $H$. Then, for $p$ odd, or $p=2$ and $k$ even,

$$
s_{k}(\text { ind } \xi)=\operatorname{tr}\left(s_{k}(\xi)\right) \text {. }
$$


For $p=2$ and $k$ odd,

$$
s_{k}(\text { ind } \xi)=\operatorname{tr}\left(s_{k}(\xi)\right)+\tau_{k},
$$

where $\tau_{k}$ is of order at most 2.

Note. Whether or not $\tau_{k}=0$ is easy to determine, but a little messy to describe.

\section{BIBLIOGRAPHY}

[At] M. Atiyah, Characters and the cohomology of finite groups, Inst. Hautes Études Sci. Publ. Math., No. 9 (1961).

[Bor] A. Borel, Topics in the homology theory of fibre bundles, Lecture Notes in Mathematics, No. 36, Springer-Verlag, New York, 1967.

[CE] H. Cartan and S. Eilenberg, Homological algebra, Princeton Univ. Press, Princeton, N. J., 1956.

[Ch1] L. Evens, On the Chern classes of representations of finite groups, Trans. Amer. Math. Soc. 115 (1965), 180-193.

[Ch2] L. Evens and D. Kahn, Chern classes of certain representations of symmetric groups, Trans. Amer. Math. Soc. 245 (1978), 309-330.

[H] F. Hirzebruch, Topological methods in algebraic geometry, Springer-Verlag, New York, 1966.

[Kn] J. Knopfmacher, On Chern classes of representations of finite groups, J. London Math. Soc. 41 (1966), 535-541.

[N] L. Evens, $A$ generalization of the transfer map in the cohomology of groups, Trans. Amer. Math. Soc. 108 (1963), 54-65.

[Q] D. Quillen, The spectrum of an equivariant cohomology ring. I, Ann. of Math. (2) 94 (1971), 549-572.

[R] F. Roush, Transfer in generalized cohomology theories, Ph.D. Thesis, Princeton Univ., 1971.

[T1] C. B. Thomas, Riemann-Roch formulae for group representations, Matematika 20 (1973), 253-262.

[T2] , An integral Riemann-Roch formula for flat line bundles, Proc. London Math. Soc. 34 (1977), 87-101.

[W] H. Weyl, The classical groups, Princeton Univ. Press, Princeton, N. J., 1946.

Department of Mathematics, Northwestern University, Evanston, Illinois 60201 